\title{
Alopecia areata update
}

\author{
Maria Hordinsky, MD and Ana Lucia Junqueira, MD
}

\section{Abstract}

There is neither a cure for alopecia areata (AA) nor any universally proven therapy that induces and sustains remission in patients afflicted with this autoimmune disease. AA is characterized as a nonscarring alopecia which affects children and adults. It can be relatively easy to treat when the disease is patchy and limited; but when children and adults present with long standing extensive scalp and body hair loss, successful management can be challenging. Of the treatment choices available, physicians and midlevel providers usually select a cost-effective treatment approach based on disease duration, disease activity, age of the patient, and disease extent. In this manuscript, the clinical presentation, epidemiology, pathophysiology, plus current and evolving treatments for AA will be reviewed

Semin Cutan Med Surg 34:72-75 (c) 2015 Frontline Medical Communications

T he most common clinical manifestations of scalp alopecia areata (AA) are either patchy or total scalp hair loss (alopecia totalis) and loss of hair along the lower occipital scalp in a band-like pattern known as ophiasis pattern AA. ${ }^{1}$ Other hairbearing regions such as the eyebrow, eyelash, facial, extremity, axillary, and pubic areas may all also be affected with AA. Alopecia universalis occurs when there is a loss of all hair fibers in all hairbearing areas.

Less typical forms of AA include sisaipho AA - which is the reverse of ophiasis pattern $\mathrm{AA}$ - with hair loss occurring on the central scalp; reticular AA is characterized by multiple patches of nonscarring hair loss interspersed with normal-appearing hair; and the diffuse variant of AA is characterized by a global decrease in scalp hair density and no discrete patches of nonscarring hair loss. Another variant is persistent patchy AA. Patients with this type of AA report ongoing active disease with patches resolving and new patches forming.

Nail plate abnormalities are commonly seen in patients with AA and range from nail pitting to severe onychodystrophy. Rarely is shedding of the nail plate reported. Likewise, ocular abnormalities have been reported but are also rarely seen., ${ }^{2,3}$

Department of Dermatology, University of Minnesota, Minneapolis, Minnesota.

Disclosures: Dr Hordinsky has performed consulting services for Incyte Corporation and Concert Pharmaceuticals. Dr Junqueira has nothing to disclose. Correspondence: Maria Hordinsky, MD; Professor and Chair; Department of Dermatology; University of Minnesota; 510 Delaware St. SE, MMC 98; Minneapolis, MN 55455. E-Mail: hordi001@umn.edu.
Examination of affected areas usually shows skin that is described as "bare as a baby's bottom". The presence of erythema, folliculitis, and/or scale should suggest another diagnosis or poor scalp hygiene. AA may also occur with other common nonscarring hair diseases such as androgenetic alopecia and telogen effluvium.

At the time of examination, AA may be either active, stable or in a regrowth phase. If lightly pulling on several fibers particularly on the scalp of patients with patchy disease is associated with easily extracted fibers, the hair-pull test is considered positive and is indicative of active disease. Close inspection of an affected area may either reveal absolutely no hair fibers, or the presence of small, lightly pigmented vellus or indeterminate fibers, or fibers which look like exclamation marks. ${ }^{4}$ If all scalp fibers are white or lightly pigmented, this may not reflect aging but rather the phenomenon of "overnight graying"; nonpigmented hair fibers are spared and only darker pigmented hair fibers are lost as part of the immune attack on the hair follicle. ${ }^{5}$ This could also be indicative of active disease affecting melanosome transfer in the hair bulb region. Dermatoscopic examination is characterized by the presence of numerous diffuse, round, and/or polycyclic yellow dots. ${ }^{6}$

AA can present with other autoimmune diseases such as thyroiditis and atopy. ${ }^{7,8}$ Multiple lines of evidence also suggest there are shared genetic risk factors between AA and other autoimmune diseases such as rheumatoid arthritis and type I diabetes. ${ }^{9}$ AA has also been found to have a considerable impact in a health-related quality of life (HRQoL). ${ }^{10}$

AA can usually be easily diagnosed. However, if the diagnosis is unclear, a scalp biopsy may be beneficial and demonstrate classic histopathologic features of AA; namely, a peribulbar lymphocytic infiltrate in a "swarm of bees" pattern, a shift in the hair cycle from the active stage of hair regrowth (anagen) to the loss phase (telogen), and dilated follicular infundibulae. ${ }^{11,12}$ Other hair disorders in the differential diagnosis include trichotillomania; traction alopecia; and tinea capitis; and less common, either secondary syphilis or loose anagen syndrome.

\section{Epidemiology}

There is no known gender predilection for AA. According to the First National Health and Nutrition Examination Survey conducted in the early 1970s, it was estimated that AA affected about 2 of every 1,000 people in the United States. ${ }^{13}$ In a 1995 study by the Mayo Clinic and the National Institutes of Health, the overall incidence of AA in Olmsted County, Minnesota was found to be 20.2 per 100,000 person-years from $1975-1989 . .^{14,15}$ In a more recent retrospective study, all patients newly diagnosed with AA from 1990 through 2009 residing in Olmsted County, Minnesota were reviewed. The cumulative lifetime incidence of AA from this more recent 20-year period (1990-2009) was 2.1\%, slightly higher than that observed in the 1995 study. ${ }^{14,15}$ 


\section{Pathophysiology}

Transplantation studies in immunodeficient mice established that T-cell immunity is involved in the pathophysiology of AA. Histologic examination of affected skin sometimes shows the hallmark "swarm of bees" around the bulb region, and an increase in the percentage of follicles in telogen. However, inflammation may be variable in long-standing extensive AA. ${ }^{11,12}$

\section{Immunology}

The normal anagen hair follicle is considered to be an immune-privileged structure as it lacks major histocompatibility complex (MHC) class I expression. ${ }^{16}$ In AA, this immune privilege is lost; and there is increased expression of both MHC class I and II antigens on the hair follicle resulting in T-cell interaction with aberrant HLADR antigens expressed by hair follicle keratinocytes. ${ }^{16,17}$ This loss of immune privilege and T-cell interaction with aberrant HLA-DR antigens expressed by hair follicle keratinocytes has been postulated to be key to the pathophysiology of AA. ${ }^{18,19}$ Although the antigen in AA remains elusive, recently cytotoxic CD8 + NKG2D + T-cells have been found to be both necessary and sufficient to induce AA; and have led to new approaches using Janus kinase (JAK) inhibitors. These and interleukin-2 (IL-2) or interleukin 15 receptor $B$ in a mouse model system have been found to be associated with a reduction in the accumulation of CD $8+\mathrm{NKG} 2 \mathrm{D}+\mathrm{T}$-cells with resultant improvement. Human trials with both IL-2 and JAK inhibitors are currently underway. ${ }^{20-22}$

\section{Genetics}

AA is considered to be a complex or multifactorial genetic trait; the familial incidence of AA is between $10 \%$ and $50 \%$. Observed heritability in first-degree relatives and twin studies further supports a genetic basis for AA. Results of candidate-gene association studies implicated associations to genes in the human leukocyte antigen (HLA) region (HLA-DQB1, HLA-DRB1, HLA-A, HLA-B, HLA$\mathrm{C}$, NOTCH4, MICA), the AIRE gene, and PTPN22. ${ }^{23}$ The results of the recently completed genome-wide association studies (GWAS) revealed at least 8 major, significant regions implicating genes of the immune system, and genes unique to the hair follicle. ${ }^{9,24,25}$ In the first GWAS study, allele frequencies were compared across nearly 500,000 single nucleotide polymorphism (SNP) markers between a group of 1,054 unrelated well-characterized AA patients primarily from the AA Registry and 3,278 population-based control subjects. A significant development was finding a strong effect of cytomegalovirus UL16-binding protein (ULBP-3) gene cluster on chromosome 6q25.1. These genes make the NKG2D-activating ligand/signal and NKG2D - an activating receptor found on natural killer cells and CD8 T cells. ${ }^{9}$

The implicated genes were also noted to associate with other autoimmune diseases such as rheumatoid arthritis, type 1 diabetes, celiac disease, and diseases with preexisting treatments including the JAK inhibitors ruxolitinib, a JAK $1 / 2$ inhibitor and tofacitinib, a JAK $1 / 3$ inhibitor. Both ruxolitinib and tofacitinib are, as of June 2015 , in clinical trials as a therapy for AA.

\section{Nervous system}

Neurogenic inflammation may also play a role in AA. Many patients with AA experience itching, tingling, formication, and/or pain with disease activity or hair regrowth. Alterations in neuro- peptide and neurotrophin expression have been reported in both humans and animal models of AA.

In studies of the expression of substance $\mathrm{P}(\mathrm{SP})$ on immune cells in the $\mathrm{C} 3 \mathrm{H} / \mathrm{HeJ}$ mouse model for alopecia areata, SP expression changed during the course of the disease, with increased expression in early stages and less in more advanced stages. ${ }^{26}$ Another neuropeptide, calcitonin gene related peptide (CGRP), has been found to be low in the plasma of affected patients. ${ }^{27}$ Changes in the expression of both SP and CGRP in immunohistochemical studies on scalp biopsy specimens have also been reported in AA as compared to normal control subjects. ${ }^{28}$ Of note, botulinum A toxin (botox) benefited one patient with cephalalgic AA. ${ }^{29}$ Additional studies to define the role of neurogenic inflammation in AA are needed.

\section{Current and evolving treatments for alopecia areata}

There is still no approved drug in the United States to treat AA; as a result, treatment options are frequently based on several parameters including: 1) Disease activity - for example, if the disease is very active, the use of systemic steroids may be warranted to halt disease activity; 2) location; 3) age of the patient; 4) disease extent; 5) assessment of a scalp biopsy with the analysis focused on the hair cycle and degree of inflammation; 6) patient expectations; 7) cost of therapy in terms of time commitment and financial resources; 8) results of screening laboratory studies ruling out the presence of other co-morbidities such as an anemia, low iron stores, thyroid abnormalities, low Vitamin D or other autoimmune diseases. Despite the inability to "cure" AA and the absence of a universally proven therapy that induces and sustains remission, many therapies are available which can benefit both children and adults with $\mathrm{AA}{ }^{30}$

\section{Current treatments}

In general, most providers treating AA patients prefer to use topical treatments to treat this disease, as in large part patients are medically healthy, and one has to consider the risks/benefits of any prescribed topical or systemic therapies. There are currently many treatment modalities available to treat AA (Table 1). However, a recent analysis of the biomedical literature database, PubMed, using the terms "randomized controlled trials" and "AA" revealed only 29 trials which strictly met these criteria. ${ }^{31}$ These studies and treatments were reviewed and analyzed using the American College of Physicians Guideline grading system and the assessment was that the majority of the studies were only of moderate quality and many had major limitations that hindered the interpretation of study results. The list of treatments can be found in Table 2. A number of treatments were found to be effective (ie, sensitizing agents such as diphenylcyclopropenone [DPCP]) and dinitrochlorobenzene [DNCB], and topical and oral corticosteroids).

Another issue with clinical research in AA is that the majority of studies have been completed in adults. In pediatric AA studies, few are controlled; in both children and adults, measuring outcomes are not usually standardized. In addition, follow-up is not included; so there is no way to prove treatment is better than placebo/natural course of the disease, particularly in patients with patchy disease. For a comprehensive review of the treatment of AA in adults a good source that is consistently updated using evidence based techniques is UptoDate. ${ }^{32}$ 
TABLE 1.Treatments for patchy and extensive alopecia areata

\author{
Patchy alopecia areata \\ - Anthralin \\ - Combination therapy \\ - Minoxidil solution-2\% or $5 \%$ \\ - Steroids in shampoo formulations \\ - Topical immunotherapy \\ - Topical or intralesional corticosteroids
}

\author{
Extensive alopecia areata \\ - Anthralin \\ - Biologics \\ - Combination therapy \\ - Corticosteroids \\ - Intralesional \\ - Oral \\ - Pulsed methlyprednisolone \\ - Topical \\ - Immunosuppressive agents \\ - Cyclosporine \\ - Methotrexate \\ - Laser therapy - Excimer laser/fractional photothermolysis \\ laser \\ - Phototherapy \\ - Narrow band ultraviolet B light \\ - Photodynamic therapy \\ - Psoralen plus ultraviolet A light \\ - Topical immunotherapy \\ - Diphenylcyclopropenone (DPCP) \\ - Squaric acid dibutylester ester (SADBE) \\ - Prostaglandin analogues \\ - Topical minoxidil
}

\section{Steroids}

Topical steroids are often the first-line approach for limited patchy AA for both children and adults. Lenane et al. demonstrated that higher potency topical steroids might be most beneficial for pediatric AA patients. Clobetasol propionate $0.05 \%$ cream and hydrocortisone $1 \%$ cream were compared in 42 patients and the investigators found the clobetasol group had a statistically significant greater amount of regrowth after 24 weeks. However, despite widespread use, there still isn't much more data in children with AA. ${ }^{33}$

The use of intralesional (IL) steroids is a mainstay of therapy of AA for adults, although the concentration and total dose that can be safely delivered continues to be debated. In our Hair Disease Clinics, we use $10 \mathrm{mg} / \mathrm{cc}$ of triamcinolone acetonide and will not inject more than $4 \mathrm{cc}$ in a session every 5 to 6 weeks. In children, the use of IL steroids is more limited but there is data from a 2002 chart review from Singapore which showed that 160/248 (65\%) of children had $>50 \%$ improvement after 12 weeks, 211/248 (85\%) with $>50 \%$ improvement after 24 weeks. ${ }^{34}$ There are also no good
TABLE 2. Medications for which randomized, controlled studies for the treatment of alopecia areata have been published

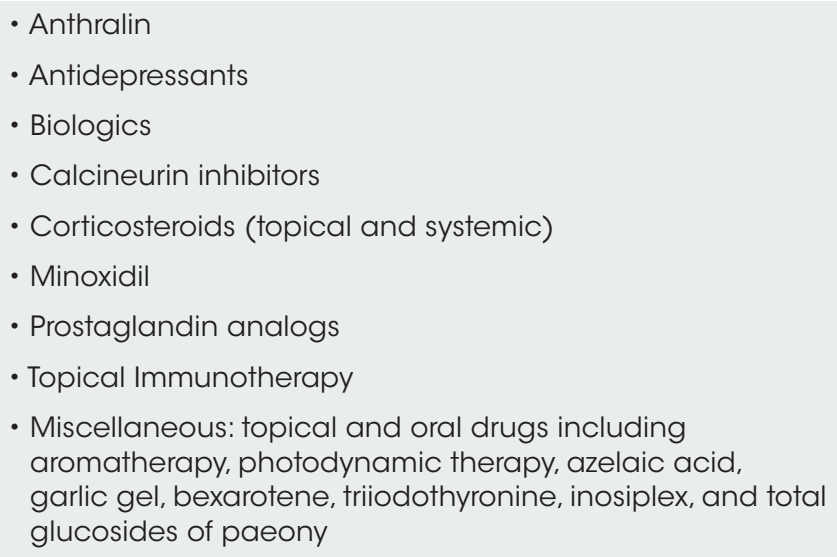

studies in children on the use of oral corticosteroids; but in the adult literature, it has been suggested that the use of prednisone is associated with a therapeutic response in about one-third of patients, one-third are steroid resistant, and the other third respond but cannot sustain a therapeutic response. ${ }^{35}$ Interestingly, both trials and chart reviews have been published on the administration of high dose pulse methylprednisolone in both children and adults. This approach may be beneficial in acute crises of hair loss. ${ }^{36}$

Topical immunotherapy may also benefit adults and children with chronic and extensive $\mathrm{AA}$, but requires frequent visits and may be more difficult for children to tolerate. Currently, there is no US Food and Drug Administration (FDA) formulation approved for use in the United States.

Management of both adult and childhood AA can include the option of "no treatment"; this holistic approach is one some patients and families desire. However, as we learn more about this autoimmune disease it is important to keep in mind that AA can associate with other autoimmune diseases, and patients may want to followup regularly for monitoring purposes.

\section{Future treatments}

Numerous treatments are available for adults and children with AA but more clinical research is needed. The findings from the first GWAS study — which led to testing JAK inhibitors in both the AA mouse model and individual human cases - and now small clinical trials have led to a new era of figuring out how effective these drugs can be; whether delivered topically or orally, in halting the AA process, growing hair, and sustaining positive clinical results. The same is true for the use of low dose IL-2 for the treatment of AA.

We are currently in an exciting era during which new treatments for AA are evolving based on the information generated from the initial GWAS study. This is particularly true for the recent excitement surrounding the use of JAK inhibitors to treat AA. At the same time, debate continues regarding the use of established therapies as well as emerging therapies in regards to the risks and benefits to young and older patients, cost of treatments, and sustainability of new and old approaches. 


\section{References}

1. Alkhalifah A, Alsantali A, Wang E, McElwee KJ, Shapiro J. Alopecia areata update: part I. Clinical picture, histopathology, and pathogenesis. J Am Acad Dermatol. 2010;62(2):177-188, quiz 189-190.

2. Tosti A, Colombati S, Caponeri GM, et al. Ocular abnormalities occurring with alopecia areata. Dermatologica. 1985;170(2):69-73.

3. Muller SA, Brunsting LA. Cataracts associated with dermatologic disorders. Arch Dermatol. 1963;88:330-339.

4. Tobin DJ. Morphological analysis of hair follicles in alopecia areata. Micross Res Tech. 1997;38(4):443-451.

5. Plinck EP, Peereboom-Wynia JD, Vuzevski VD, Westerhof W, Stolz E. Turning white overnight, is it possible? [in Dutch] Ned Tijdschr Geneeskd. 1993;137(42):12071210

6. Miteva M, Tosti A. Hair and scalp dermatoscopy. J Am Acad Dermatol. 2012;67(5):1040-1048.

7. Goh C, Finkel M, Christos PJ, Sinha AA. Profile of 513 patients with alopecia areata: associations of disease subtypes with atopy, autoimmune disease and positive family history. J Eur Acad Dermatol Venereol. 2006;20(9):1055-1060.

8. Hordinsky MK. Overview of alopecia areata. J Investig Dermatol Symp Proc. 2013;16(1):S13-S15.

9. Petukhova L, Duvic M, Hordinsky M, et al. Genome-wide association study in alopecia areata implicates both innate and adaptive immunity. Nature. 2010;466(7302):113-117.

10. Colón EA, Popkin MK, Callies AL, Dessert NJ, Hordinsky MK. Lifetime prevalence of psychiatric disorders in patients with alopecia areata. Compr Psychiatry. 1991;32(3):245-251.

11. Möller CS, El Shabrawi-Caelen L. Follicular Swiss cheese pattern-another histopathologic clue to alopecia areata. J Cutan Pathol. 2011;38(2):185-189.

12. Whiting DA. Histopathologic features of alopecia areata: a new look. Arch.Dermatol. 2003;139(12):1555-1559.

13. Safavi K. Prevalence of alopecia areata in the First National Health and Nutrition Examination Survey. Arch Dermatol. 1992;128(5):702.

14. Safavi KH, Muller SA, Suman VJ, Moshell AN, Melton LJ $3^{\text {rd }}$. Incidence of alopecia areata in Olmsted County, Minnesota, 1975 through 1989. Mayo Clin Proc. 1995;70(7):628-633.

15. Mirzoyev SA, Schrum AG, Davis MD, Torgerson RR. Lifetime incidence risk of alopecia areata estimated at $2.1 \%$ by Rochester Epidemiology Project, 1990-2009. J Invest Dermatol. 2014;134(4);1141-1142.

16. Ito $\mathrm{T}$. Recent advances in the pathogenesis of autoimmune hair loss disease alopecia areata. Clin Dev Immunol. 2013;2013:348546.

17. Gilhar A, Paus R, Kalish RS. Lymphocytes, neuropeptides, and genes involved in alopecia areata. J Clin Invest. 2007;117(8):2019-2027.

18. Kang $\mathrm{H}, \mathrm{Wu} \mathrm{WY}$, Lo BK, et al. Hair follicles from alopecia areata patients exhibit alterations in immune privilege-associated gene expression in advance of hair loss. J Invest Dermatol. 2010;130(11):2677-2680.

19. Paus R, Nickoloff BJ, Ito T. A ‘hairy’ privilege. Trends Immunol. 2005;26(1):32-40.
20. Xing L, Dai Z, Jabbari A, et al. Alopecia areata is driven by cytotoxic T lymphocytes and is reversed by JAK inhibition. Nature Med. 2014;20(9):1043-1050.

21. Craiglow B, King BA. Killing two birds with one stone: oral tofacitinib reverses alopecia universalis in a patient with plaque psoriasis. $J$ Invest Dermatol. 2014;134(12):2988-2990.

22. Castela E, Le Duff F, Butori C, et al. Effects of low-dose recombinant interleukin 2 to promote T-regulatory cells in alopecia areata. JAMA Dermatol. 2014;150(7):748751

23. Jabbari A, Petukhova L, Cabral RM, Clynes R, Christiano AM. Genetic basis of alopecia areata: a roadmap for translational research. Dermatol Clin. 2013;31(1):109 117.

24. Jagielska D, Redler S, Brockschmidt FF, et al. Follow-up study of the first genomewide association scan in alopecia areata: IL13 and KIAA0350 as susceptibility loci supported with genome-wide significance. J Invest Dermatol. 2012;132(9):21922197.

25. Forstbauer LM, Brockschmidt FF, Moskvina V, et al. Genome-wide pooling approach identifies SPATA5 as a new susceptibility locus for alopecia areata. Eur $J$ Hum Genet. 2012;20(3):326-332.

26. Siebenhaar F, Sharov AA, Peters EM, et al. Substance P as an immunomodulatory neuropeptide in a mouse model for autoimmune hair loss (alopecia areata). $J$ Invest Dermatol. 2007;127(6):1489-1497.

27. Daly TJ. Alopecia areata has low plasma levels of the vasodilator/immunomodulatory calcitonin gene-related peptide. Arch Dermatol. 1998;134(9):1164-1165.

28. Hordinsky M, Ericson M. Autoimmunity: alopecia areata. J Investig Dermatol Symp Proc. 2004; 9(1):73-78.

29. Cutrer FM, Pittelkow MR. Cephalalgic alopecia areata: a syndrome of neuralgiform head pain and hair loss responsive to botulinum A toxin injection. Cephalalgia. 2006;26(6):747-751.

30. Delamere FM, Sladden MM, Dobbins HM, Leonardi-Bee J. Interventions for alopecia areata. Cochrane Database Syst Rev. 2008;(2):CD004413.

31. Hordinsky M, Donati A. Alopecia areata: an evidence-based treatment update. Am J Clin Dermatol. 2014;15(3):231-246.

32. UpToDate. Management of alopecia areata. Messenger AG, Dellavalle RP, Hordinsky M, Ofori AO, eds. http://www.uptodate.com/contents/management-of-alopeciaareata? source $=$ search result\&search $=$ Messenger + AG. + Management + of + alopecia + areata\&selectedTitle $=1 \sim 150$. Accessed June 16, 2015.

33. Lenane P, Macarthur C, Parkin PC, et al. Clobetasol propionate, $0.05 \%$, vs hydrocortisone, $1 \%$, for alopecia areata in children: a randomized clinical trial. JAMA Dermatol. 2014;150(1):47-50.

34. Tan E, Tay YK, Giam YC. A clinical study of childhood alopecia areata in Singapore. Pediatr Dermatol. 2002; 19:298-301.

35. Winter RJ, Kern E, Blizzard RM. Prednisone therapy for alopecia areata. Arch Dermatol. 1976;112(11):1549-1552.

36. Sharma VK, Muralidhar S. Treatment of widespread alopecia areata in young patients with monthly oral corticosteroid pulse. Pediatr Dermatol. 1998;15(4): 313-317. 